\title{
Nationwide Survey of Acute Juvenile Female Non-Herpetic Encephalitis in Japan: Relationship to Anti-N-Methyl-D-Aspartate Receptor Encephalitis
}

\author{
Satoshi Kamei ${ }^{1}$, Shigeki Kuzuhara ${ }^{2}$, Masaki Ishihara ${ }^{1}$, Akihiko Morita ${ }^{1}$, Naoto Taira ${ }^{1}$, \\ Masaki Togo ${ }^{1}$, Makoto Matsui ${ }^{3}$, Masafumi Ogawa ${ }^{2}$, Kinya Hisanaga ${ }^{4}$, \\ Tomohiko Mizutani ${ }^{1}$ and Sadako Kuno ${ }^{2}$
}

\begin{abstract}
Objective To study the incidence and clinical features of acute juvenile female non-herpetic encephalitis (AJFNHE) in Japan.

Methods A nationwide questionnaire on patients with severe non-herpetic encephalitis of unknown etiology with a prolonged clinical course or death was sent to the departments of Internal Medicine, Neurology, Pediatrics, and Emergency and Critical Care at all hospitals with 200 beds or more in Japan.

Results The recovery rate was 25\% (1,279 out of 5,030 departments) and 90 patients were enrolled in this study. The annual incidence was $0.33 / 10^{6}$ population. $85 \%$ of patients were female. The means and standard deviations of age at onset and hospital stay were $26 \pm 10$ years and $180 \pm 228$ days. As first symptoms, fever and psychosis were presented in $90 \%$. Among the neurological symptoms, disturbance of consciousness was presented in $92 \%$, convulsions in $65 \%$, and involuntary movements in 55\%. Respiratory failure during hospitalization was observed in $71 \%$ and required care with mechanical ventilation. The detection rate of antiGluR $\varepsilon 2$ and/or $\delta 1$ antibodies was $67 \%$ of patients. Anti-N-methyl-D-aspartate receptor NR1/NR2 antibody was detected in all four examined patients with anti-GluR $\varepsilon 2$ antibody, and also detected in both of the two examined patients without anti-GluR $\varepsilon 2$ antibody. As for outcome, $46 \%$ returned to work and $37 \%$ returned home, but $7 \%$ died. Associated tumors were demonstrated in 39\%. All reported patients had ovarian tumors, among which teratoma was the most frequent.
\end{abstract}

Conclusion A nationwide survey provided data for the annual incidence and clinical features of AJFNHE in Japan.

Key words: incidence, clinical features, acute juvenile female non-herpetic encephalitis (AJFNHE), anti-Nmethyl-D-aspartate receptor (NMDAR) encephalitis

(Inter Med 48: 673-679, 2009)

(DOI: 10.2169/internalmedicine.48.1898)

\section{Introduction}

In 1997, Nishimura et al (1) first reported five young adult female patients with acute non-herpetic encephalitis who presented with severe prolonged coma and status epilepticus, but achieved a good recovery. Following this report, the results of a clinical analysis on 89 serial patients with encephalitides indicated that 11 such patients presented specific and different clinical features, including the frequent

\footnotetext{
${ }^{1}$ Division of Neurology, Department of Medicine, Nihon University School of Medicine, Tokyo, ${ }^{2}$ Department of Neurology, National Center Hospital of Neurology and Psychiatry, Kodaira, ${ }^{3}$ Department of Neurology, Kanazawa Medical University, Ishikawa and ${ }^{4}$ Department of Neurology, Miyagi National Hospital, Miyagi

Received for publication November 27, 2008; Accepted for publication January 20, 2009

Correspondence to Dr. Satoshi Kamei, skamei@med.nihon-u.ac.jp
} 
Table 1. Regional Differences in Annual Incidence of the Registered Patients

\begin{tabular}{lcc}
\hline \multicolumn{1}{c}{ Regions } & Annual incidence $\left(/ 10^{6}\right.$ population $)$ & Regional differences \\
\cline { 1 - 2 } Hokkaido & 0.39 & \\
\cline { 1 - 2 } Tohoku & 0.43 & \multirow{2}{*}{ non-significant } \\
\cline { 1 - 2 } Kanto & 0.25 & \\
\hline Chubu-Hokuriku & 0.20 & \\
\hline Kinki-Chugoku & 0.50 & \\
\hline Kyikoku & 0.36 & \\
\hline
\end{tabular}

detection of anti-glutamate receptor (GluR) antibody as compared with other etiologies of encephalitis. The specific clinical features of such patients demonstrated severe encephalitis with a prolonged clinical course and the negative result of herpes simplex virus infection, but these patients achieved a relatively good recovery. Since all of their 11 patients were young adult women, we designated these patients as "acute juvenile female non-herpetic encephalitis (AJFNHE)" (2).

In 2007, Dalmau et al (3) reported anti-N-methyl-Daspartate receptor (NMDAR) encephalitis associated with ovarian teratoma. Based on the similarity of clinical features in both kinds of patients, Iizuka $\mathrm{T}$ et al (4) recently described the detection of anti-NMDAR antibody in four patients who were clinically diagnosed as having AJFNHE; moreover, in three of these four patients, ovarian teratoma was confirmed.

Here, we describe the results of a nationwide survey on AJFNHE in Japan, including the relationships between AJFNHE and anti-NMDAR encephalitis.

\section{Methods}

A nationwide questionnaire on the incidence and clinical features in patients with severe encephalitis of unknown etiology with a prolonged clinical course or death, who were also confirmed to have a negative result for herpes simplex virus infection, was sent for completion to the 5,030 departments of Internal Medicine, Neurology, Pediatrics, and Emergency and Critical Care at all hospitals with 200 beds or more in Japan during a period from April 2004 to March 2007. A prolonged clinical course was defined as no improvement of their neurological symptoms during the period of more than one month after onset. The criteria of AJFNHE in the present study did not thus include the sex of patients, age of onset, nor the MRI finding. The questionnaire on clinical features in such patients was also sent to all members of the Research Group for Comprehensive Clinical Studies on the Diagnosis, Treatment and Prevention of Neurological Disorders (Chairman: Sadako Kuno, M.D., National Center Hospital of Neurology and Psychiatry) during the period from April 1987 to March 2007. The overlap of the registered patients in the present study was excluded based on detailed history of their admission and transfer.

After confirmation of the above-mentioned criteria in each patient, we evaluated the following factors: annual incidence and regional differences in incidence, sex, age of onset, du- ration of hospitalization, clinical symptoms, respiratory failure, laboratory data, treatment, outcome, and associated tumors. In addition, we analyzed the data of GluR antibodies and anti-NMDAR antibody.

The annual incidence per $10^{6}$ population was estimated on the basis of the number of registered patients and the total population of Japan in 2005, and regional differences in incidence were analyzed by the Ryan test (5). Differences in clinical features between female and male patients were assessed by Fisher's exact probability test or the MannWhitney U test. SPSS statistical software Version 12.0 (SPSS Inc., Chicago, Illinois) was employed for the statistical analysis. The level of significance for this study was defined as 0.05 .

\section{Results}

The questionnaire recovery rate was $25 \%$ (1,279 out of 5,030 departments), and the questionnaires of 129 patients returned for this nationwide survey. 90 out of these 129 patients were enrolled in the present study based on the abovementioned criteria. The excluded 39 patients consisted of 12 patients with the encephalopathy associated with influenza virus infection, 5 patients with human herpes virus 6 encephalitis, 2 patients with acute disseminated encephalomyelitis, 10 patients without data confirming negative result of herpes simplex virus infection, and 10 patients without the prolonged clinical course of more than one month after onset. A total of 44 of 90 patients were encountered during the period from April 2004 to March 2007. Therefore, the annual incidence and regional differences in incidence were estimated based on the data of 44 patients, the other analyses were estimated based on the data of 90 patients.

\section{Estimated annual incidence and regional differences}

The estimated annual incidence in Japan was $0.33 / 10^{6}$ population. The annual incidences in each region ranged from 0.20 to 0.50 per $10^{6}$ population and the regional differences was found to be non-significant (Table 1).

\section{Gender}

Among the enrolled patients, $85 \%$ were female. This form of encephalitis thus predominantly affected females, although it also occurred in male patients.

\section{Age of onset and duration of hospitalization}

The mean and standard deviation (SD) of age of onset 
A. Age of onset

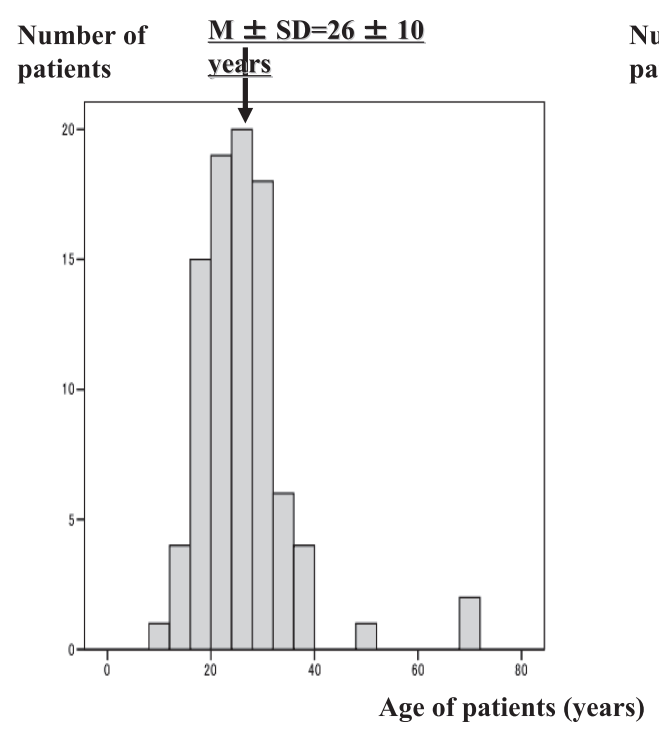

\section{B. Duration of hospitalization}

Number of

patients

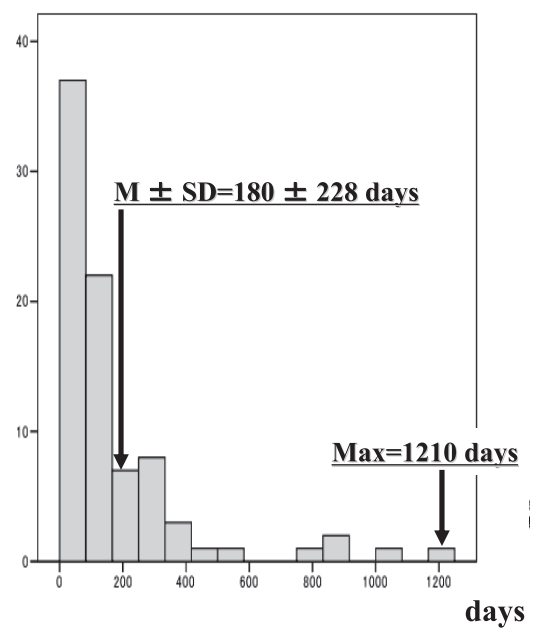

Figure 1. Distribution of age of onset (A) and duration of hospitalization (B). M: mean value, Max: maximum value, SD: standard deviation

was $26 \pm 10$ years. The mean and SD of length of hospital stay was $180 \pm 228$ days, and the maximum was 1,210 days (Fig. 1).

\section{Clinical symptoms}

A prodrome of cold-like symptoms was observed in $90 \%$ of enrolled patients. As first symptoms, fever and psychosis were presented in $90 \%$ of patients. Concerning the vital signs on admission, the mean and SD of body temperature was $37.7 \pm 0.9^{\circ} \mathrm{C}$, that of systolic blood pressure was $123 \pm 18$ $\mathrm{mmHg}$, and that of respiration was $20 \pm 6.7$ per min. Among the neurological symptoms observed during the entire clinical course, disturbance of consciousness was demonstrated in $92 \%$ of patients, fluctuations of blood pressure in $78 \%$, convulsions in $65 \%$, and involuntary movements in $55 \%$. The distribution of the duration from onset to initial improvement of the neurological findings was ranged from 31 to 730 days, and the mean and SD value was $149 \pm 160$ days (Fig. 2).

\section{Respiratory failure}

Respiratory failure on admission was observed in $30 \%$ of patients, but care with mechanical ventilation during hospitalization was required in $78 \%$ of patients. Among the patients with care with mechanical ventilation, $91 \%$ suffered central respiratory failure without respiratory infection and the remaining $9 \%$ received mechanical ventilation because of general anesthesia due to control seizures. Central hypoventilation during the entire of clinical course was demonstrated in $71 \%$ of patients. The mean and SD of the duration of care with mechanical ventilation was $102 \pm 153$ days and the maximum value was 933 days (Fig. 3).

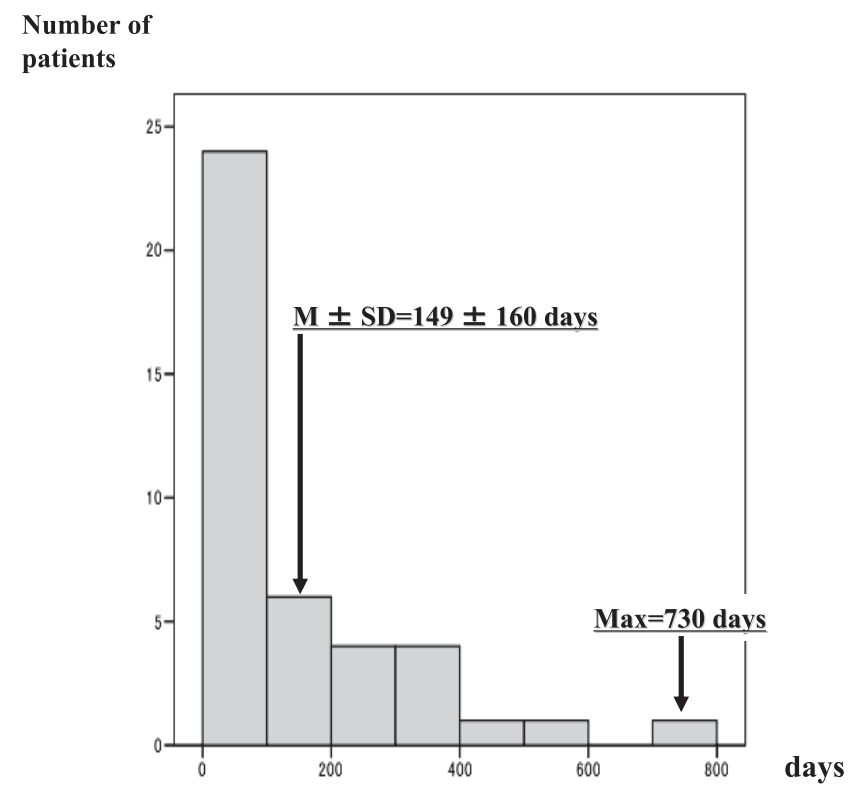

Figure 2. Distribution of duration from onset to initial improvement of neurological findings. M: mean value, Max: maximum value, $\mathrm{SD}$ : standard deviation

\section{Laboratory data}

The mean value and SD of the cell count in the initial cerebrospinal fluid (CSF) on admission was $71 \pm 112 / \mu \mathrm{L}$, and that of the protein concentration was $50 \pm 54 \mathrm{mg} / \mathrm{dL}$. The EEG findings revealed that a diffuse slow and/or paroxysmal wave was present in $89 \%$ of patients. Concerning the findings of cranial MRI, $74 \%$ of patients demonstrated normal findings and the remaining $26 \%$ revealed medial temporal lobe lesions.

Regarding anti-GluR antibody, the anti-NMDAR GluR $\varepsilon 2$ and $\delta 1$ antibodies, which were measured by Dr. Yukitoshi 
Number of

patients

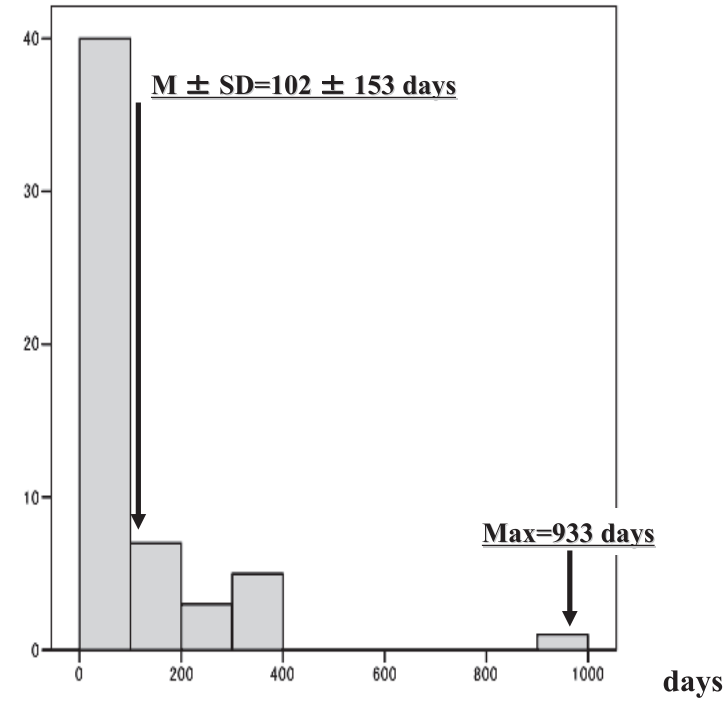

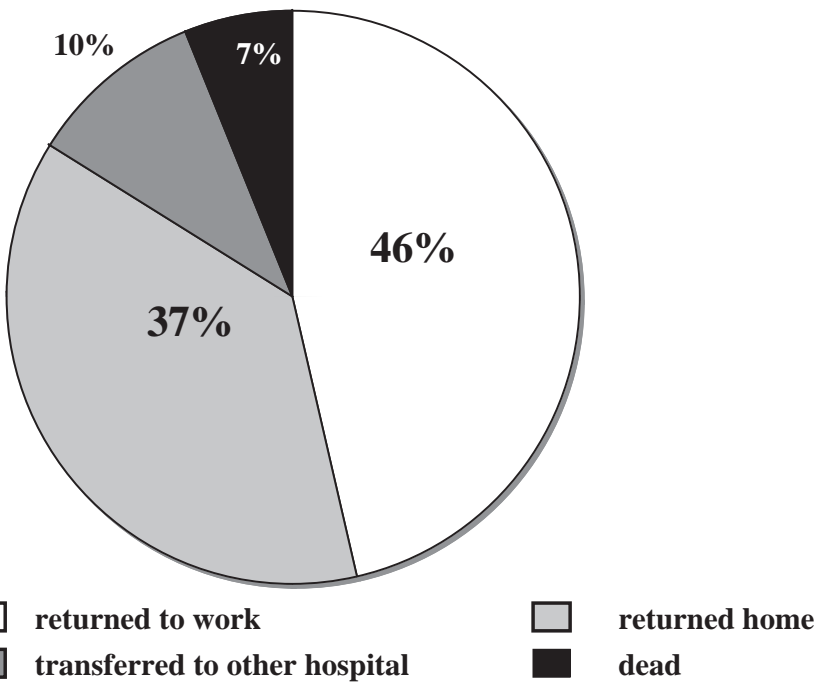

Figure 4. Outcome of registered patients with AJFNHE. AJFNHE: acute juvenile female non-herpetic encephalitis

Figure 3. Distribution of duration of care with mechanical ventilation. $M$ : mean value, Max: maximum value, $\mathrm{SD}$ : standard deviation

Table 2. Detailed Positive Rates of Anti-GluR Antibodies which Have a Linear Epitope to a Single Subunit of NMDAR

\begin{tabular}{|c|c|c|c|c|c|c|c|c|}
\hline \multirow[b]{3}{*}{ Sample } & \multicolumn{4}{|c|}{ Anti-GluR $\varepsilon 2$ antibody } & \multicolumn{4}{|c|}{ Anti-GluR $\delta 1$ antibody } \\
\hline & \multicolumn{2}{|c|}{ Serum } & \multicolumn{2}{|c|}{ CSF } & \multicolumn{2}{|c|}{ Serum } & \multicolumn{2}{|c|}{ CSF } \\
\hline & $\mathrm{IgG}$ & IgM & $\mathrm{IgG}$ & $\operatorname{IgM}$ & $\operatorname{IgG}$ & IgM & $\mathrm{IgG}$ & $\operatorname{IgM}$ \\
\hline $\begin{array}{c}\text { Numbers of } \\
\text { examined patients }\end{array}$ & 15 & 17 & 20 & 19 & 14 & 14 & 19 & 19 \\
\hline $\begin{array}{l}\text { Numbers of } \\
\text { patients with } \\
\text { positive result }\end{array}$ & 3 & 8 & 7 & 4 & 4 & 5 & 5 & 5 \\
\hline Positive rate (\%) & 20 & 47 & 35 & 21 & 29 & 36 & 26 & 26 \\
\hline
\end{tabular}

$\mathrm{CSF}=$ cerebrospinal fluid; GluR $=$ glutamate receptor; $\mathrm{NMDAR}=\mathrm{N}$-methyl-D-aspartate receptor

Takahashi (6), their data were available in 24 patients, and the rate of detection of either of these antibodies in the serum, CSF or both samples was $67 \%$ of patients (16 out of the 24 patients examined). The detailed results for the positive rates of GluR $\varepsilon 2$ and $\delta 1$ antibodies (Table 2) showed that the rate of GluR $\varepsilon 2 \operatorname{IgM}$ antibody in the serum was $47 \%$, and that of GluR \&2 IgG in the CSF was 35\%. On the other hand, anti-NMDAR NR1/NR2 heteromer antibody, which was measured by Dr. Josep Dalmau (3), was detected in all out of the four examined patients who had also revealed anti-NMDAR GluR \&2 antibody. Moreover, antiNMDAR NR1/NR2 heteromer antibody was further detected in both of the two examined patients who presented negative results for anti-NMDAR GluR $\varepsilon 2$ antibody.

\section{Outcome and treatment}

Data on outcome demonstrated that $46 \%$ of patients returned to work, and $37 \%$ returned home, but $7 \%$ of patients died (Fig. 4). The patients with the present form of encephalitis had a severe and prolonged clinical course, but their outcome in the long term was relatively good. The rate of patients with sequelae was $52 \%$ of the survived patients. The observed sequelae consisted of cognitive dysfunction in $39 \%$ of patients with sequelae, and psychosis and epilepsy in $23 \%$ each.

The treatments included anti-viral drugs in $89 \%$ of patients, corticosteroids in $83 \%$, intravenous immunoglobulin in $32 \%$, and plasma exchange in 3\%. Moreover, antiepileptic drugs were administered in $94 \%$ of patients, and intravenous anesthetics in $49 \%$. Overall, half of the patients required intravenous administration of anesthetics in order to control their convulsions.

\section{Associated tumors}

Associated tumors were demonstrated in $23(39 \%)$ of the 59 examined patients, ovarian tumors were found in all 23 patients. The types of associated tumor in the 23 patients consisted of teratoma in 17 patients, ovarian cyst in two patients, ovarian cancer in one patient, a history of ovarian tumor in two patients, and not described in one patient. Tera- 
Table 3. Differences in Clinical Features between Female and Male Patients

\begin{tabular}{|c|c|c|c|}
\hline & $\begin{array}{c}\text { Females } \\
\left(68 \text { patients }{ }^{\#}\right)\end{array}$ & $\begin{array}{c}\text { Males } \\
\left(12 \text { patients }^{\#}\right)\end{array}$ & $\begin{array}{c}\text { Difference* of } \\
\text { clinical features } \\
\text { between female and } \\
\text { male patients }\end{array}$ \\
\hline Age of onset (years) [mean \pm SD] & $24.5 \pm 9.0$ & $31.3 \pm 13.4$ & $\mathrm{p}=0.026^{*}$ \\
\hline Duration of hospitalization (days) [mean $\pm \mathrm{SD}]$ & $179 \pm 222$ & $143 \pm 238$ & NS \\
\hline Prodromes & $91 \%$ & $82 \%$ & NS \\
\hline \multicolumn{4}{|l|}{ First symptoms } \\
\hline Fever & $90 \%$ & $83 \%$ & NS \\
\hline Psychosis & $93 \%$ & $73 \%$ & NS \\
\hline \multicolumn{4}{|l|}{ Vital signs on admission } \\
\hline Body temperature $\left({ }^{\circ} \mathrm{C}\right)[$ mean $\pm \mathrm{SD}]$ & $37.6 \pm 0.8$ & $37.7 \pm 0.8$ & NS \\
\hline Systolic blood pressure $(\mathrm{mmHg})[$ mean $\pm \mathrm{SD}]$ & $125.1 \pm 17.9$ & $130.7 \pm 16.1$ & NS \\
\hline Respiration $(/ \mathrm{min})[$ mean $\pm \mathrm{SD}]$ & $20.0 \pm 7.5$ & $19.8 \pm 5.2$ & NS \\
\hline \multicolumn{4}{|l|}{ Neurological symptoms } \\
\hline Disturbance of consciousness & $91 \%$ & $92 \%$ & NS \\
\hline Convulsions & $67 \%$ & $67 \%$ & NS \\
\hline Involuntary movements & $54 \%$ & $50 \%$ & NS \\
\hline Mechanical ventilation & $78 \%$ & $75 \%$ & NS \\
\hline Diffuse slow and/or paroxysmal wave on EEG & $90 \%$ & $92 \%$ & NS \\
\hline $\begin{array}{ll}\text { MRI findings } & \begin{array}{l}\text { normal } \\
\text { abnormal (site; medial temporal lobe) }\end{array}\end{array}$ & $\begin{array}{l}71 \%, \\
29 \%\end{array}$ & $\begin{array}{l}67 \%, \\
33 \%\end{array}$ & NS \\
\hline Outcome & $\begin{array}{l}\text { improved } 95.6 \% \\
\text { dead } 4.4 \%\end{array}$ & $\begin{array}{l}\text { improved } 75 \% \\
\text { dead } 25 \%\end{array}$ & $\mathrm{p}=0.041^{*}$ \\
\hline $\begin{array}{l}\text { Positive rate of anti-GluR antibody in serum and/or CSF samples } \\
\text { (positive numbers of patients / total numbers of examined } \\
\text { patients) }\end{array}$ & $\begin{array}{c}68 \% \\
(15 / 22)\end{array}$ & $\begin{array}{l}50 \% \\
(1 / 2)\end{array}$ & NS \\
\hline $\begin{array}{l}\text { Associated tumor } \\
\text { (numbers of patients with associated tumor / total numbers } \\
\text { of examined patients) }\end{array}$ & $49 \%(20 / 41)^{\# \#}$ & $0 \%(0 / 10)$ & $\mathrm{p}=0.004^{*}$ \\
\hline
\end{tabular}

toma was thus the most frequent.

Moreover, we attempted to contact the 14 registered patients including 11 previously reported patients (2) in the present study who had been treated at the Division of Neurology, Department of Medicine, Nihon University School of Medicine, to undergo further examinations for ovarian teratoma. Three patients could not be contacted because they moved to another town. Ten $(91 \%)$ out of the remaining 11 patients demonstrated ovarian teratoma by MRI or had a history of a removal operation for ovarian tumor. Among the 11 previously reported patients (2), the eight $(89 \%)$ out of nine patients except for two patients, who were impossible to have contact, demonstrated ovarian teratoma or had a history of a removal operation for ovarian tumor.

\section{Differences in clinical features between female and male patients}

The clinical features in male patients with the present encephalitis were almost identical to those in female patients, except for slightly older age of onset and somewhat higher mortality rate, and the absence of associated tumor including teratoma (Table 3).

\section{Discussion}

A comparison of clinical features between the enrolled patients with AJFNHE in the present study and the reported patients with anti-NMDAR encephalitis (3) (Table 4) indicated that both patient groups showed almost identical clinical features. Most of the patients were female, and young adults were predominantly affected. A prodrome was presented in over $80 \%$ of patients. The first neurological symp- tom was psychosis in over $90 \%$ of patients. Convulsions, disturbance of consciousness, and involuntary movements were presented as the main neurological symptoms during the entire clinical course. Mechanical ventilation was required in about $80 \%$ of patients. Regarding outcome, 93\% of patients in this survey were improved, whereas Dalmau et al (3) reported that $75 \%$ of patients were improved in spite of marked severe condition at acute stage. Concerning the MRI findings, the frequency of medial temporal lesion was about one-fourth of patients.

The detection of GluR $\varepsilon 2$ or $\delta 1$ antibodies in this survey was $67 \%$ of examined patients, whereas Dalmau et al (3) reported the NMDAR NR1 and NR2 heteromer antidody was detected in all 12 patients. The GluR $\varepsilon 2$ antibody, which was measured by Yukitoshi Takahashi (6), and the NMDAR NR1/NR2 heteromer antibody, which was measured by Josep Dalmau (3), are both antibodies to NMDA type glutamate receptor. The GluR $\varepsilon 2$ antibody has a linear epitope which recognizes N-terminal regions in the NR2B subunit. The binding region of such an antibody is thus a single subunit. On the other hand, the NMDAR NR1/NR2 antibody has a conformational epitope which recognizes the NR 1 and NR2 heteromers. This difference in epitopes might be reflected in the difference of frequency of positive antibodies in patients with such encephalitis.

Dalmau et al (3) reported that ovarian teratoma was demonstrated in 11 out of 12 patients, and mediastinal teratoma in one patient, whereas associated tumors were demonstrated in $23(39 \%)$ of the 59 examined patients in this survey; ovarian tumors were found in all 23 patients. The period of this nationwide survey just preceded the publication of Dalmau's report (3), and an association with ovarian teratoma 
Table 4. Comparison of Clinical Features between the National Survey of AJFNHE in the Present Study and the Reported Patients with Anti-NMDAR Encephalitis

\begin{tabular}{|c|c|c|c|}
\hline & $\begin{array}{l}\text { National survey of } \\
\text { AJFNHE } \\
\text { in the present study } \\
90 \text { patients }\end{array}$ & $\begin{array}{l}\text { Anti-NMDAR encephalitis } \\
12 \text { patients } \\
\text { (Dalmau J et al: } 2007 \text { ) }\end{array}$ & $\begin{array}{l}\text { Difference* of clinical } \\
\text { features between the } \\
\text { two studies }\end{array}$ \\
\hline Females & $85 \%$ & $100 \%$ & NS \\
\hline $\begin{array}{l}\text { Age of onset (years; mean } \pm \\
\text { SD) }\end{array}$ & $26 \pm 10$ & $28 \pm 9$ & NS \\
\hline Prodrome & $90 \%$ & $83 \%$ & NS \\
\hline First neurological symptom & psychosis $(90 \%)$ & $\begin{array}{c}\text { psychosis }(75 \%) \\
\text { memory loss } \rightarrow \text { psychosis }(25 \%)\end{array}$ & NS \\
\hline Convulsions & $65 \%$ & $92 \%$ & NS \\
\hline Disturbance of consciousness & $92 \%$ & $100 \%$ & NS \\
\hline Involuntary movements & $55 \%$ & $67 \%$ & NS \\
\hline Mechanical ventilation & $78 \%$ & $83 \%$ & NS \\
\hline Outcome & improved $93 \%$, dead $7 \%$ & improved $75 \%$, dead $25 \%$ & NS \\
\hline $\begin{array}{l}\text { Frequency of medial temporal } \\
\text { lesion using MRI }\end{array}$ & $26 \%$ & $25 \%$ & NS \\
\hline Antibody (positive \%) & $\begin{array}{l}\text { GluR } \varepsilon 2 \text { or } \delta 1 \\
\qquad(67 \%)\end{array}$ & $\begin{array}{l}\text { NMDAR NR1/NR2 heteromer } \\
\qquad(100 \%)\end{array}$ & $\mathrm{p}=0.03$ \\
\hline Associated tumor & $\begin{array}{l}\text { Ovarian tumors were found } \\
\text { in all } 23 \text { patients with } \\
\text { associated tumor. }\end{array}$ & $\begin{array}{l}\text { Ovarian teratoma; } 11 \text { patients } \\
\text { Mediastinal teratoma; } 1 \text { patient }\end{array}$ & $\begin{array}{c}\text { NS } \\
\text { (Frequency of ovarian } \\
\text { tumor) }\end{array}$ \\
\hline
\end{tabular}

*The differences were assessed by Fisher's exact probability test or Mann-Whitney $U$ test.

AJFNHE=acute juvenile female non-herpetic encephalitis; $\mathrm{GluR}=$ glutamate receptor; $\mathrm{MRI}=$ magnetic resonance imaging; $\mathrm{NMDAR}=$

$\mathrm{N}$-methyl-D-aspartate receptor, $\mathrm{NS}=$ not significant

in such encephalitis thus could not be well recognized in Japan. However, the results for associated tumors of AJFNHE in the present study indicated that ovarian teratoma was the most frequent. Moreover, it was also able to confirm the presence of ovarian teratoma using MRI or a history of a removal operation for ovarian tumor in $91 \%$ of patients including Kamei's previously reported patients (2) in the present study. Therefore, AJFNHE and anti-NMDAR encephalitis were inferred to be almost identical condition. AJFNHE represented a clinical concept based on the specific clinical features, and anti-NMDAR encephalitis represented a clinical entity based on the neuro-oncological findings including the NMDAR NR1 and NR2 heteromer antibody.

Their clinical features in this nationwide survey on AJFNHE were uniform and also in concordance with those previously reported as AJFNHE (2). The present nationwide survey of AJFNHE undertaken in Japan revealed the following: the annual incidence was $0.33 / 10^{6}$ population; there were no regional differences in the incidence; respiratory failure was observed in about $70 \%$ of patients and required care with mechanical ventilation; associated tumors were demonstrated in about $40 \%$ of patients, and ovarian teratoma was the most frequent; and male patients with such encephalitis were also registered and their clinical features were very similar to those of female patients except for the absence of associated tumor.

There has been a short description of male patients with anti-NMDAR NR1/NR2 encephalitis as unpublished data in a recently reported review (7). Such encephalitis was therefore considered to affect not only female patients with related ovarian teratoma, but also male patients. Based on a consideration of the association with teratoma, mediastinal teratoma and seminoma might be examined in male patients with such encephalitis.

However, several problems still remain to be unresolved including the etiology of patients without anti-NMDAR antibody, the etiology of male patients, the lack of detectable ovarian teratomas in some patients, the reason why predominantly young adults are affected, the reason why many Japanese patients are reported $(4,8-10)$, and the reason why there is a good outcome in the long term without tumor removal.

\section{Conclusion}

The patients collected in our nationwide survey of AJFNHE were predominantly young adult women. Their clinical features were uniform and also in concordance with those previously reported as AJFNHE except for the presence of small number of male patients. The survey data revealed that the annual incidence was $0.33 / 10^{6}$ population without regional differences, respiratory failure was observed in about $70 \%$ and required care with mechanical ventilation, associated tumors were demonstrated in about $40 \%$, ovarian teratoma was the most frequent, and the clinical feature of male patients was very similar to that of female patients except for the absence of associated tumor.

\section{Acknowledgement}

We are indebted to Ichiro Kanazawa, M.D., President of the Science Council of Japan, and Toshiaki Takasu, M.D., Professor Emeritus of Nihon University, Tokyo, Japan, for their support regarding the present nationwide survey, and to Yukitoshi Takahashi, M.D., Director of the National Epilepsy Center, Shizuoka 
Institute of Epilepsy and Neurological Disorders, Shizuoka, Japan, and Josep Dalmau, M.D., Professor of the Division of Neuro-Oncology, Department of Neurology, University of Pennsylvania, Philadelphia, PA, USA, for the measurement of glutamate receptor antibodies. We are also grateful to all participating doctors for their cooperation in the nationwide survey.

The present study was performed as one of the works of the
Research Group for Comprehensive Clinical Studies on the Diagnosis, Treatment and Prevention of Neurological Disorders, which was supported by a Research Grant (18A-9) for Nervous and Mental Disorders from the Ministry of Health, Labour and Welfare, Japan, and by a Grant from the Ministry of Education, Culture, Sports, Science, and Technology of Japan for the promotion of research foundation, Japan.

\section{References}

1. Nishimura T, Miki K, Ogawa K, et al. Pathogenesis of aseptic acute non-herpetic encephalitis: young adult female patients with acute non-herpetic encephalitis who presented with severe prolonged coma, but achieved a good recovery. Neuroinfection 2: 7476, 1997 (in Japanese).

2. Kamei S. Acute juvenile female non-herpetic encephalitis (AJFNHE). Adv Neurol Sci 48: 827-836, 2004.

3. Dalmau J, Tüzün E, Wu HY, et al. Paraneoplastic anti-N-methylD-aspartate receptor encephalitis associated with ovarian teratoma. Ann Neurol 61: 25-36, 2007.

4. Iizuka T, Sakai F, Ide T, et al. Anti-NMDA receptor encephalitis in Japan: long-term outcome without tumor removal. Neurology 70: 504-511, 2008.

5. Ryan TA. Significance tests for multiple comparison of proportions, variances, and other statistics. Psychol Bull 57: 318-328, 1960 .
6. Takahashi Y, Mori H, Mishina M, et al. Autoantibodies to NMDA receptor in patients with chronic forms of epilepsia partialis continua. Neurology 61: 891-896, 2003.

7. Tüzün E, Dalmau J. Limbic encephalitis and variants: classification, diagnosis and treatment. Neurologist 13: 261-271, 2007.

8. Seki M, Suzuki S, Iizuka T, et al. Neurological response to early removal of ovarian teratoma in anti-NMDAR encephalitis. J Neurol Neurosurg Psychiatry 79: 324-326, 2008.

9. Okamoto K, Yamazaki T, Banno H, Sobue G, Yoshida M, Takatama M. Neuropathological studies of patients with possible non-herpetic acute limbic encephalitis and so-called acute juvenile female non-herpetic encephalitis. Intern Med 47: 231-236, 2007.

10. Miyazaki M, Yoshino A, Teraishi T, Nomura S, Nemoto H, Takahashi Y. Encephalitis of unknown etiology with anti-GluR $\varepsilon 2$ autoantibody, showing divergent neuroradiologic and clinical findings. Eur Neurol 57: 111-113, 2007.

(C) 2009 The Japanese Society of Internal Medicine http://www.naika.or.jp/imindex.html 